\title{
SYNERGISTIC EFFECT OF COMBINED TREATMENT WITH EXTRA VIRGIN OLIVE OIL AND DOXORUBICIN ON SQUAMOUS CELL CARCINOMA CELL LINE
}

\author{
Hala El-Kammar* and Shaimaa Eliwa Ghazy**
}

\begin{abstract}
Background: In spite of the fact that the medical care has been extremely improved, the survival rate of patients diagnosed with Squamous cell carcinoma is still decreased . Doxorubicin (DXR) is one of the most effective anti- squamous cell carcinoma drugs. This drug has a dangerous side effects. Natural products can lighten the DXR- induced side effects, without compromising its antitumor efficacy. The consumption of extra virgin olive oil (EVOO), appeared to be related to fewer malignant tumor occurrences. Aim of the study: The objective of the present study was to evaluate the effect of EVOO when used alone and, more interestingly, when combined with DXR, for 48 hours, on lingual squamous cell carcinoma cell line (SCC25). Materials and methods: SCC25 was used in this study, it was divided into four groups. Group I (control), group II (DXR treated), group III (EVOO treated) and group IV (EVOO+DXR treated). Results: there was significant decrease in MMP-1, VEGFR-2 expressions as well as migration and invasion between the test groups and the control group. Conclusion: EVOO is a promising anticancer compound when used alone or in combination with DXR.
\end{abstract}

KEYWORDS: Squamous cell carcinoma, Doxorubicin, olive oil, matrix metalloproteinase, vascular endothelial growth factor receptor.

\section{INTRODUCTION}

The most common malignant tumor in the oral cavity is Squamous cell carcinoma of the tongue (SCCOT). Because of the high lymphatic drainage of the tongue tissue, distant metastasis and a high death rates are commonly occurring, at the time of diagnosis of SCCOT. In spite of the fact that the medical care has been extremely improved, the survival rate is still decreased with a disappointing cure rate ${ }^{(1,2)}$.

Many researches have focused on cytotoxic drugs a lot. Although there is a great improvement in clarifying the pathogenesis of cancer, surgical removal of tumors beside cytotoxic drugs and radiation still remain the most influential treatment choice. Chemoprevention depends on synthetic,

\footnotetext{
* Lecturer of Oral Pathology, Faculty of Oral and Dental Medicine, Future University in Egypt

** Lecturer of Oral Pathology, Faculty of Dentistry, Ain Shams University
} 
semisynthetic or natural drugs to restrain the progress of cancer $^{(3)}$. Doxorubicin (DXR) is considered a successful anti-cancer agents, it is used usually in combination with other cytotoxic agents for the treatment of the different tumor types such as oral squamous cell carcinoma ${ }^{(4,5)}$.

However, this drug has a dangerous side effects, such as cardiac toxicity. As a consequence, the use of DXR as a cytotoxic drug has some restrictions. These limitations made it very critical to search for other agents that can lighten the DXR-induced side effects, without affecting its antitumor effect ${ }^{(4,6)}$.

Natural compounds act pleiotropically on cancer cells. They are able to modulate some of the signaling pathways involved in carcinogenesis, like the antiproliferative, pro-apoptotic, and anti-angiogenic pathways ${ }^{(7)}$. The amount and quality of consumed fat, has been involved in cancer occurrence, either positively or negatively ${ }^{(2)}$.

The intake of extra virgin olive oil (EVOO), which is a famous habit of the Mediterranean population, appeared to have a strong relation to a lesser occurrence of many malignant tumors. The effect of olive oil on human life has mainly been investigated in Mediterranean countries, where it is used in large amounts. Its fatty acid component is mainly oleic acid, followed by palmitic and linoleic acids ${ }^{(8)}$. In addition, the extra-virgin olive oil contains phenolic antioxidants, like simple phenols, aldehydic secoiridoids, flavonoids and lignans ${ }^{(2,7,9,10)}$. Nevertheless, it is not known till now which components are responsible for its valuable effects ${ }^{(11,12)}$

Many steps are needed for metastasis to occur, such as cell detachment, invasion, proliferation and neo-vascularization ${ }^{(13,14)}$. Interactions of tumor cells with the extracellular matrix are important events in the growth and invasion of malignancy ${ }^{(7,15)}$.

Matrix metalloproteinases (MMPs), a group of Zn2+-dependent endogenous proteases, are capable of breaking down different ingredients of the extracellular matrix. In the extracellular domain, the per- formance of these proteases is controlled by inhibitors known as tissue inhibitors of metalloproteinases (TIMPs) ${ }^{(16,17)}$. The imbalance between MMPs and TIMPs may be used as a prognostic indicator for cancer. The over expression of MMP-1 in oral SCC promotes angiogenesis and accelerates oral SCC tumorigenesis and invasion ${ }^{(17-19)}$.

New blood vessels formation is a critical step in tumor growth and invasion. Studies have shown that the expression of vascular endothelial growth factor (VEGF) associates with tumor invasion and metastasis. VEGF signals are transmitted intracellularly by binding with class III tyrosine kinase receptors, known as vascular endothelial growth factor receptors (VEGFRs). Studies concerning VEGFR-receptors expression in OSCCs revealed that all three receptors (VEGFR-1, VEGFR-2, and VEGFR-3) were consistently expressed on tumor cells and vascular endothelial cells, along with a predominant overexpression of VEGFR-2 ${ }^{(20,21)}$. More over other studies also demonstrated that, MMPs increase the bioavailability of VEGFR, which results in neovascularization $^{(17,22,23)}$.

The objective of the present study was to evaluate the effect of EVOO when used alone and, more interestingly, when combined with DXR, for 48 hours, on lingual Squamous cell carcinoma cell line (SCC25)

\section{MATERIAL AND METHODS}

\section{The material used in this study}

Cell line: SCC25 cells of the American Type Culture Collection (ATCC)® were obtained from VACSERA-EGYPT. The cells were grown in RPMI 1640 containing $10 \%$ fetal bovine serum at $37^{\circ} \mathrm{C}$.

Doxorubicin: the drug was purchased in powder form, in a $10 \mathrm{mg}$ bottle with mw.579.98, from Sigma-Aldrich ${ }^{\circledR}$

Extra virgin olive oil extract: Extra virgin olive oil from GAWDA ${ }^{\circledR}$, Benghazi, Libya 


\section{Methods}

\section{Inverted phase contrast microscope}

The samples were viewed under the Inverted Phase Contrast Microscope (Olympus CKX41)®, for evaluation of confluence of the SCC25 cell line in the studied groups.

\section{MTT assay for the determination of the IC-50}

The viability of the tested cells in all groups was established using the MTT (3-[4,5-dimethylthiazol2-yl]-2.5-diphenyl tetrazolium bromide) in vitro toxicology assay kit, MTT based, Stock No. TOX1 (Trevigen SIGMA, Saint Louis, Missouri, USA) as per manufacturer's protocol. Briefly, cells were plated in a 96-well tissue culture plates in a range of $103-105$ cells/well in a final volume of $100 \mathrm{uL}$ of the medium and were allowed to attach overnight.

The MTT reagent was applied (10 ul per well) and the plate was incubated for $12 \mathrm{~h}$ to promote intracellular reduction of the soluble yellow MTT to the insoluble purple formazan dye. The detergent reagent was applied to each well to solubilize the formazan dye before evaluation of the absorbance of each sample in a ROBONIK P2000 Spectrophotometer, wave length:450-560 $\mathrm{nm}$. Using the MTT assay, the IC50 for each substance was calculated and summarized in table (1). All the experiments there-after were performed using the calculated IC50 for 48 hours. duration for each group.

TABLE (1) The IC-50 for the DXR, EVOO and DXR+EVOO

\begin{tabular}{|c|c|}
\hline The substance tested & IC50 ug/ml(SCC25) \\
\hline DXR & $2.49 \pm 0.36$ \\
\hline EVOO & $64.31 \pm 4.36$ \\
\hline DXR+EVOO & $0.78 \pm 0.059$ \\
\hline
\end{tabular}

\section{Quantitative Sandwich immunoassay- ELIZA technique}

For detection of each of the proteins, Human ELISA Kit was used, concerning the VEGFR-2 kit, it was purchased from RayBio-Cyman chemicals $\AA$ and the MMP-1 kit was purchased from SigmaAldrich $\AA$. Briefly, the following procedure was repeated for each of the proteins. A 96-well plate was coated with an antibody specific for each of the studied proteins. Standards and samples were pipetted into the wells and the protein existing in the sample is attached to the wells via the immobilized antibody. The wells were washed and biotinylated anti-human antibody was applied. After discarding of the unbound biotinylated antibody, horseradish peroxidase enzyme (HRP) conjugated streptavidin was pipetted to the wells. The wells were washed again, a 3,3',5,5'-Tetramethylbenzidine (TMB) substrate solution was applied to the wells and color developed was attributed to the amount of protein bound. The Stop Solution changed color from blue to yellow, and the intensity of the color is measured at $450 \mathrm{~nm}$.

\section{Cell invasion and migration assay (CORNING $\left.{ }^{\circledR}\right)$}

SCC25 cells were grown in RPMI 1640 containing $10 \%$ fetal bovine serum at $37^{\circ} \mathrm{C}$,

\section{Migration Assay}

The migration assay was done with the chemotactic directional migration by using a 96 well Transwell Permeable Supports with $8 \mu \mathrm{m}$ pores (Corning Cat. No.3374). The cells (104 cells/well in serum-free media) from all groups were placed in the upper chamber, whereas the lower chamber was filled with media supplemented with $10 \%$ FBS as a chemoattractant. After incubation for 24 hours of, the cells that did not migrate in the upper chamber were separated using a cotton swab then the filters were stained independently with $0.2 \%$ crystal 
violet $(600 \mu \mathrm{l})$ and the migrated cells adherent to the bottom of the filter were inspected, calculated and photographed with a microscope at 200x magnification.

\section{Invasion Assay}

The invasion assay was performed by using coated -96 well Transwell Permeable Supports with $8 \mu \mathrm{m}$ pores (Corning Cat. No.3374) coated with basement membrane extract purchased from (Trevigen cat. No. 3455-096-02). The cells from all groups (104 cells/well) were applied in the upper compartment of the transwell insert. The inferior compartment was filled with DMEM supplemented with $10 \%$ of FBS. The cells were incubated for 24 hours $\left(37^{\circ} \mathrm{C}\right.$, humidified atmosphere, $95 \%$ air and $5 \% \mathrm{CO} 2$ ). Finally, cells were stained as above described.

\section{Statistical Analysis}

The collected data were tabulated using Microsoft Excel (Microsoft Office 2007). All cases were included in the statistical analysis. The data were tabulated using the Statistical Package for Social Science (SPSS 15.0) Software. The mean values and standard errors were calculated for each time point from the pooled normalized data. The statistical tests performed included the One Way ANOVA and Post Hoc test for comparison of means. The results were considered significant when the $\mathrm{P}$ value was $\leq 0.05$. Graphs were performed using Microsoft powerpoint software (Microsoft Office 2007).

\section{RESULTS}

\section{Inverted phase contrast microscopic results}

The number of cells appeared to be decreased in all three tested groups (figs 1b,1c and 1d) compared to the control group (fig 1a) which showed the most confluent appearance.

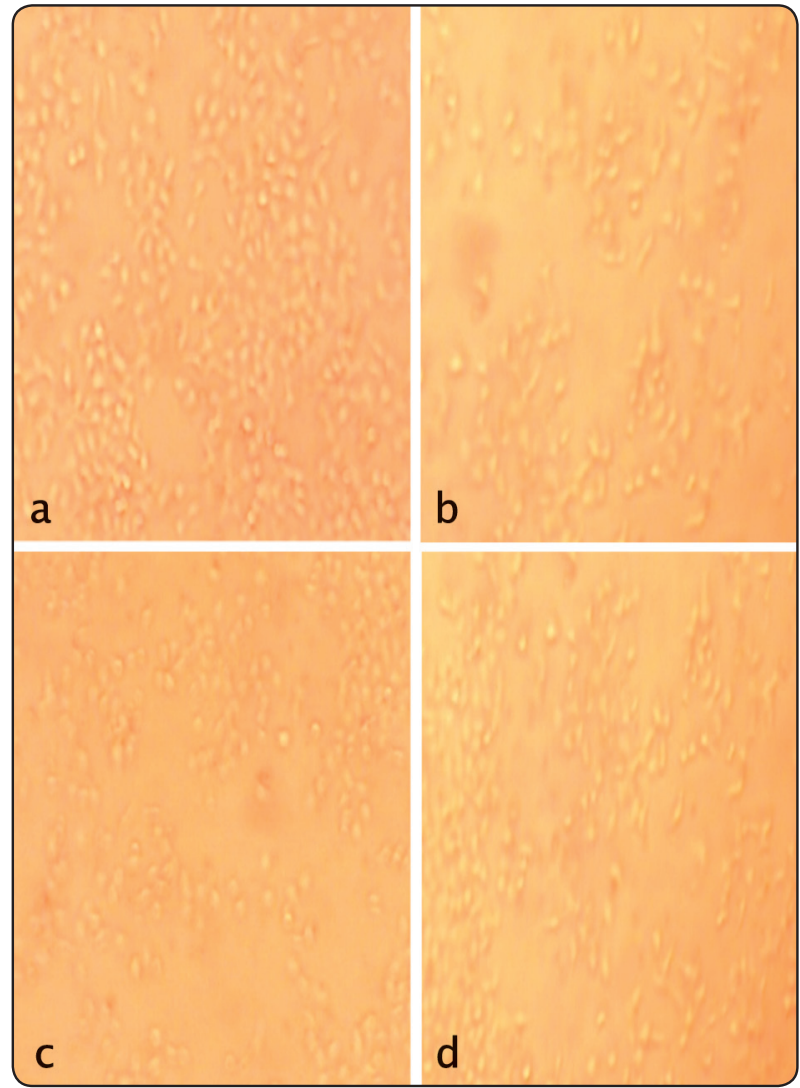

Fig 1: Photomicrograph of the studied groups at IC50. Fig 1a: photomicrograph of the control group (group I). Fig 1b: photomicrograph of the DXR-treated group (group II). Fig 1c: photomicrograph of the EVOO treated group (group III). Fig1d: photomicrograph of group IV (DXR+EVOO). (orig. mag. X20)

\section{Statistical results}

\section{ELIZA, invasion and migration assays results}

One way ANOVA (table 2), revealed that there was a significance difference in mean MMP-1, VEGFR-2 protein values and invasion and migration assay values, in the studied groups.

TABLE (2) One Way ANOVA for the studied groups

\begin{tabular}{|c|c|c|}
\hline Test & $\mathrm{F}$ & Sig. \\
\hline MMP-1 & 148.598 & .000 \\
\hline VEGFR-2 & 40818.821 & .000 \\
\hline Invasion & 1411.313 & .000 \\
\hline Migration & 201819.371 & .000 \\
\hline
\end{tabular}


The highest MMP-1 and VEGFR-2 expressions were observed in group I (control) $(=10550$ and 5360 , respectively) (table 3 and fig 2). The difference in MMP-1 and VEGFR-2 mean expression in the control group and any of the tested groups was significant, using the Post Hoc test (table 5)

(Table 3 and fig 2) revealed that MMP1 expression was lowest in group II (= 2264), followed by group IV (=3896), and the difference between them was significant $(\mathrm{p}=0.000)$, using the Post Hoc test (Table 5), while the least VEGFR-2 expression was noted in group IV $(=220)$, followed by Group II (=238) and the difference between them was insignificant.

From (Table 4), it was noted that the highest percentages of invasion and migration were in the group I (=4.3 and=1.92, respectively), Using the Post Hoc test, the difference between the control group and any of the test groups, regarding invasion and migration, was always highly significant $(\mathrm{p}=0.00)$ (table 5). The least invasion percentage was noted in group IV $(=1.17)$ followed by group II and finally group III (table 4), with no significant difference between all tested groups, using the Post Hoc test (table 5).

Regarding the migration percentages, the least value was noted in group III $(=0.58)$ Followed by group IV $(=0.63)$ and finally group II (table 4$)$. The differences in migration percentages among all experimental groups were always insignificant, except for the difference in migration between groups II and III, which was significant $(\mathrm{p}=0.016)$, using the Post Hoc test (table 5).

TABLE (3) Descriptive analysis of the MMP-1 and VEGFR-2 protein values in the studied groups

\begin{tabular}{|c|c|c|c|}
\hline Protein & Groups & Mean & SD \\
\hline \multirow{4}{*}{ MMP-1 } & Control & 10550.0000 & .81650 \\
\cline { 2 - 4 } & DXR & 2264.0000 & 166.46922 \\
\cline { 2 - 4 } & EVOO & 4733.0000 & 312.36944 \\
\cline { 2 - 4 } & DXR+EVOO & 3896.0000 & 149.36979 \\
\hline \multirow{5}{*}{ VEFGR-2 } & Control & 5360.0000 & .81650 \\
\cline { 2 - 4 } & DXR & 238.5000 & 2.03142 \\
\cline { 2 - 4 } & EVOO & 1050.0000 & 21.49419 \\
\cline { 2 - 4 } & DXR+EVOO & 220.0000 & 3.55903 \\
\hline
\end{tabular}

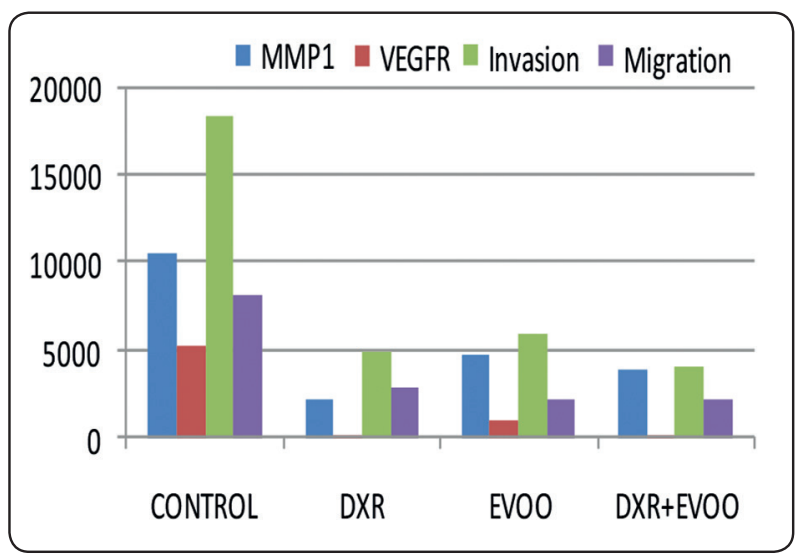

Fig. (2) Bar chart showing the mean protein expression by ELISA of MMP-1 and VEGFR-2 and the mean invasion and migration assay values in the studied groups. 
TABLE (4) Descriptive analysis of the invasion and migration assay results in the studied groups

\begin{tabular}{|c|c|c|c|c|c|c|c|}
\hline Group & Total Count & Invasion Count & \% Invasion & SD & Migration Count & \% Migration & SD \\
\hline Control & 428000 & 18404 & 4.3 & .21602 & 8217.6 & 1.92 & .05888 \\
\hline DXR & 359000 & 4990.1 & 1.39 & .24644 & 2836.1 & 0.79 & .09345 \\
\hline EVOO & 376000 & 5940.8 & 1.58 & .28705 & 2180.8 & 0.58 & .10863 \\
\hline DXR+EVOO & 347000 & 4059.9 & 1.17 & .02160 & 2186.1 & 0.63 & .02944 \\
\hline
\end{tabular}

TABLE (5) Post hoc (Pairwise analysis) for MMP-1 and VEGFR-2 protein values and invasion and migration assay values among the studied groups

\begin{tabular}{|c|c|c|c|c|c|}
\hline \multirow{2}{*}{\multicolumn{2}{|c|}{ Compared Groups }} & \multicolumn{4}{|c|}{ p-value } \\
\hline & & MMP-1 & VEGFR-2 & Invasion $\%$ & Migration \% \\
\hline Control & DXR & $.000 *$ & $.000 *$ & $.000 *$ & $.000 *$ \\
\hline Control & EVOO & $.000 *$ & $.000 *$ & $.000 *$ & $.000 *$ \\
\hline Control & DXR+EVOO & $.000 *$ & $.000 *$ & $.000 *$ & $.000 *$ \\
\hline DXR & EVOO & $.000 *$ & $.000 *$ & 1.000 & $.016^{*}$ \\
\hline DXR & DXR+EVOO & $.000 *$ & .205 & 1.000 & .085 \\
\hline EVOO & DXR+EVOO & $.000 *$ & $.000 *$ & .125 & 1.000 \\
\hline
\end{tabular}

$P \leq 0.05$

\section{DISCUSSION}

The treatment of SCCOT has been a great challenge and considered as a debate because of its increased rate of metastasis and bad prognosis ${ }^{(1)}$. In the present study doxorubicin (DXR) anticancer drug was used. DXR has a definite cytotoxic impact on the human oral squamous cell carcinoma cell lines compared to normal human mesenchymal normal oral cells, indicating high tumor-specificity. Recently however,DXR was found to have powerful cytotoxicity against normal human keratinocytes of the oral mucous membrane ${ }^{(4)}$.

As a result, the research effort has been directed to identify effective drugs or strategies that can reduce DXR toxicity without decreasing its antitumor efficacy, that's why we selected EVOO as it a natural plant that has been proved to have a cytotoxic behavior aganist many cancer types such as bladder, breast and colon cancer ${ }^{(6,7,9)}$. However, to our knowledge, the effect of EVOO on oral cancer has not been investigated sufficiently. For this reason this study evaluates the potential anticarcingenic effect of EVOO on SCCOT cell line when used alone or when combined with DXR and to explore some of the molecular mechanism behind its effect.

In the present study both DXR and EVOO showed an inhibitory effect on SCCOT cells proliferation using the MTT assay after 48 hours. 
Co-treatment with both reagents, showed an additive inhibition of cell proliferation at the same duration. These findings are of great importance, as addition of EVOO to DXR, is capable of lowering the concentration of DXR while obtaining a more significant anti-proliferative effect on the SCCOT cells, thereby, decreasing the side effects of DXR. This result is in agreement with Papachristodoulou A, et al, 2016 (24), who revealed that addition of EVOO extract with DXR showed a powerful sensitization of DXR mediated cytotoxicity on prostate cancer cells. The cytotoxic effect of EVOO may be attributed to either its fatty acid fraction such as oleic acid which allows cell cycle arrest in G0G1 phase through lowering the cell cycle regulator CyclinD1 and allows the apoptotic pathway through the obvious inhibition of the anti-apoptotic protein Bcl-2 and enhancement of the expression of the proapoptotic proteins caspase- $3^{(2,11,15)}$.

The other proposed mechanism by which EVOO exerts its cytotoxic effect, may be through its polyphenolic compounds, such as oleocanthal and oleuropein ${ }^{(12)}$. Oleuropein prevents cell proliferation by arresting the cell cycle at $\mathrm{S}$ phase and increasing the cyclin-dependent inhibitor $\mathrm{p} 21^{(9)}$. Moreover, it was found to down-regulate the oncogenic proteins $\mathrm{NF}-\mathrm{\kappa B}$ and cyclin D1 leading to reduction of cell proliferation, while oleocanthal was found to have a cytotoxic effect through downregulation of Bcl-2, Akt, and ERK1/2 in melanoma ${ }^{(15)}$.

Metastasis is considered the major obstacle in the cancer therapy and it is responsible for bad prognosis and also has a direct effect on the survival rate of patients. In this study in vitro cell migration and invasion assays were selected in order to investigate whether EVOO has an anti-metastatic effect on SCCOT cells ${ }^{(16)}$.

The main advantages of the in-vitro assays are their relatively easy handling and their being less expensive than the in vivo assays. Yet, it should mention here, that none of the used assays could exactly simulate every fundamental step of metastasis, but they just examine parts of it. For the present study, the Transwell migration and invasion assay was selected, as this assay is the commonly used to investigate cell migration and invasion properties in-vitro $^{(16,25)}$.

The results of this study, demonstrated a significant decrease in migration and invasion percentages of OSSCT cells in all tested groups compared to the control, so the use of EVOO alone or mixed with DXR has a significant antimetastatic property against OSSCT. These results were consistent with previous studies which revealed that EVOO has anti-metastatic effect on colon and bladder cancer cells. This could be explained by the fact that EVOO polyphenols such as Oleocanthal, block the activation of STAT3. This ultimately downregulats its downstream effectors, including the epithelial mesenchymal transition (EMT) transcriptional factors, Twist and MMP family $(3,10,26)$. EMT has been suggested to play a prominent role in the occurrence of a migration and invasion phenotype of many carcinoma cells ${ }^{(27)}$.

The lowest invasion percentage in this study, was seen in group IV (DXR + EVOO) followed by group II (DXR) and finally group III (EVOO) with no statistical difference in between them confirming the anti -invasive effect of EVOO when added to DXR or when used alone. Concerning the migration assay, the lowest migration percentage was observed in group III (EVOO) followed by group IV (DXR+EVOO) and finally group II (DXR)and there was only a statistical significant difference between group II and III, this could be explained by either that EVOO might have more anti-migratory effect on OSSCT than DXR and this requires further investigation about all aspects involved in migration, as we mentioned before that in vitro assays investigate only a part of the whole process.

Another explanation that is worth mentioning here, is the fact that epithelial cells, can show various migration patterns. Epithelial cells are mostly stationary, tightly packed, nevertheless, 
during development they can be mobilized and through wound repair. Epithelial cells can either migrate in the form of sheets, tubular structures, or as individual cells ${ }^{(17,28)}$.

Collective migration is different from single cell migration mainly in that cells remain connected throughout the process, maintaining intact cell-cell junctions through movement and finally exhibiting migratory patterns that are primarily distinct from the migratory patterns of single cells ${ }^{(14)}$. Unfortunately the transwell migration assay used in the present study measures only the single cell migration ${ }^{(16,25)}$.

Also, Haris R., et al, 2009, mentioned that certain anticancer drugs at suitable concentrations were capable of selectively stop certain types of migration. Single cell migration was selectively inhibited by paclitaxel while deoxyuridine exclusively inhibited the collective type of migration and the borrelidin drug was able to stop both types of cancer cell migration. So we would recommend future investigations with different types of cell migration assays that measure the collective cell migration such as wound healing migration assay in order to confirm the action of both compounds on the different types of cell migration ${ }^{(29)}$.

In order to explore the molecular mechanism by which both DXR and EVOO exert their antimigratory and invasive effect we selected to investigate expression of MMP-1 which has been reported to be associated with OSCC development and its expression increases with poor histolgical $\operatorname{grades}^{(30)}$. The role of MMPs in tumorigenesis and angiogenesis has been confirmed by many authors during the past few decades ${ }^{(7,18)}$.

This study revealed a significant decrease in the MMP-1 expression in all tested groups compared to the control group, this was in agreement with other researches who proved that EVOO modulates the MMP family expression through its polyphenols components that decrease the metastatic potential by inhibiting cell migration and invasion which is mediated by MPP family ${ }^{(7,8,31)}$.
The lowest value of MMP-1 expression observed in group II (DXR), this result was in accordance with Benbow U. et al ,1999, who reported that DXR particularly inhibited MMP-1 gene expression in the aggressive metastatic human melanoma cell line ${ }^{(32)}$.

The second lowest value of MPP-1 was observed in group IV (DXR+EVOO) and the difference between it and group II was significant, while group III (EVOO) showed a higher expression of MMP-1 compared to the rest of the tested groups this could be explained by that many members of MMPs family (other than MMP-1) are involved in progression of SSCOT such as MMP-2, MMP-7 MMP-9 and MMP-13 ${ }^{(18)}$, so the EVOO might act on other subtypes of MMP rather than MMP-1 and this needs further investigations in order to clarify the exact effect of EVOO on the different MMPs family members.

Angiogenesis is considered one of the most important factors in tumor progression and metastasizing potential of solid tumors. VEGF stimulates crucial signaling processes that regulate tumor angiogenesis and, therefore, represents an another influential target for the development of novel anticancer agents ${ }^{(20,21)}$.

The present study revealed a statistically significant decrease of the VEGFR-2 expression in all tested groups, compared to the control group, with the least value for mean expression in group IV (DXR+ EVOO), followed by group II (DXR) and then group III (EVOO). So, it can be deducted that the use of EVOO exerts the highest inhibitory effect on VEGFR-2 expression when added to DXR with no statistical difference between group II (DXR) and IV (DXR+EVOO). This finding is in good agreement with, who demonstrated that olive oil is a potent inhibitors of the VEGFR-2 signaling pathway either through its fatty acid component or its polyphenols through its inhibitory effect on specific autophosphorylation sites of VEGFR-2 
(Tyr951, Tyr1059, Tyr1175 and Tyr1214) leading to the inhibition of endothelial cell (EC) signaling. These findings emphasize the chemopreventive properties of olive oil and highlight the importance of nutrition in cancer prevention(20).

\section{CONCLUSION}

The results of the present study provide evidence that EVOO has a very promising cytotoxic effect on SCCOT when used alone or when introduced in a co-treatment scheme with DXR.

EVOO has a clear anti-migratory, anti-invasive and anti-angiogenic properties when used on its own or with DXR.

EVOO potentiates the anti-carcinogenic effect of DXR at a lower dose.

\section{RECOMMENDATION}

Future investigations are needed to explore the in vivo efficacy of EVOO in animal models and explore more information of its molecular mechanism of action.

\section{REFERENCES}

1. Azimi H, Khajehahmadi S, Rahpeyma A. Tongue squamous cell carcinoma: A Clinical Study. Iranian Journal of Pathology. 2014;9(1):28-32.

2. Psaltopoulou T, Kosti RI, Haidopoulos D, Dimopoulos M, Panagiotakos DB. Olive oil intake is inversely related to cancer prevalence: a systematic review and a meta-analysis of 13800 patients and 23340 controls in 19 observational studies. Lipids in health and disease. 2011;10(1):127.

3. Lewandowska U, Gorlach S, Owczarek K, Hrabec E, Szewczyk K. Synergistic interactions between anticancer chemotherapeutics and phenolic compounds and anticancer synergy between polyphenols. Advances in Hygiene \& Experimental Medicine/Postepy Higieny i Medycyny Doswiadczalnej. 2014;68.

4. Sakagami H, Okudaira N, Masuda Y, Amano O, Yokose S, Kanda Y, et al. Induction of apoptosis in human oral keratinocyte by doxorubicin. Anticancer research. 2017;37(3):1023-9.
5. Khiavi MM, Rostami A, Hamishekar H, Abassi MM, Aghbali A, Salehi R, et al. Therapeutic efficacy of orally delivered doxorubicin nanoparticles in rat tongue cancer induced by 4-nitroquinoline 1-oxide. Advanced pharmaceutical bulletin. 2015;5(2):209.

6. Minotti G, Menna P, Salvatorelli E, Cairo G, Gianni L. Anthracyclines: molecular advances and pharmacologic developments in antitumor activity and cardiotoxicity. Pharmacological reviews. 2004;56(2):185-229.

7. Coccia A, Bastianelli D, Mosca L, Monticolo R, Panuccio I, Carbone A, et al. Extra virgin olive oil phenols suppress migration and invasion of T24 human bladder cancer cells through modulation of matrix metalloproteinase-2. Nutrition and cancer. 2014;66(6):946-54.

8. Dell'Agli M, Fagnani R, Galli GV, Maschi O, Gilardi F, Bellosta $\mathrm{S}$, et al. Olive oil phenols modulate the expression of metalloproteinase 9 in THP-1 cells by acting on nuclear factor- $x \mathrm{~B}$ signaling. Journal of agricultural and food chemistry. 2010;58(4):2246-52.

9. Elamin MH, Daghestani MH, Omer SA, Elobeid MA, Virk P,Al-Olayan EM, et al. Olive oil oleuropein has anti-breast cancer properties with higher efficiency on ER-negative cells. Food and chemical toxicology. 2013;53:310-6.

10. Owen R, Giacosa A, Hull W, Haubner R, Spiegelhalder B, Bartsch $H$. The antioxidant/anticancer potential of phenolic compounds isolated from olive oil. European Journal of Cancer. 2000;36(10):1235-47.

11. Jiang L, Wang W, He Q, Wu Y, Lu Z, Sun J, et al. Oleic acid induces apoptosis and autophagy in the treatment of Tongue Squamous cell carcinomas. Scientific Reports. 2017;7(1):11277.

12. Cicerale S, Conlan XA, Barnett NW, Sinclair AJ, Keast RS. Influence of Heat on Biological Activity and Concentration of Oleocanthal a Natural Anti-inflammatory Agent in Virgin Olive Oil. Journal of agricultural and food chemistry. 2009;57(4):1326-30.

13. Bozzuto G, Ruggieri P, Molinari A. Molecular aspects of tumor cell migration and invasion. Annali dell'Istituto superiore di sanit Â. 2010;46:66-80.

14. Valencia AMJ, Wu P-H, Yogurtcu ON, Rao P, DiGiacomo J, Godet I, et al. Collective cancer cell invasion induced by coordinated contractile stresses. Oncotarget. 2015;6(41):43438.

15. Fogli S, Arena C, Carpi S, Polini B, Bertini S, Digiacomo $\mathrm{M}$, et al. Cytotoxic activity of oleocanthal isolated from virgin olive oil on human melanoma cells. Nutrition and cancer. 2016;68(5):873-7. 
16. Kramer N, Walzl A, Unger C, Rosner M, Krupitza G, Hengstschläger $M$, et al. In vitro cell migration and invasion assays. Mutation Research/Reviews in Mutation Research. 2013;752(1):10-24.

17. Friedl P, Wolf K. Tumour-cell invasion and migration: diversity and escape mechanisms. Nature Reviews Cancer. 2003;3(5):362.

18. Mishev G, Deliverska E, Hlushchuk R, Velinov N, Aebersold D, Weinstein F, et al. Prognostic value of matrix metalloproteinases in oral squamous cell carcinoma. Biotechnology \& biotechnological equipment. 2014;28(6):1138-49.

19. Gakiopoulou H, Nakopoulou L, Siatelis A, Mavrommatis I, Panayotopoulou EG, Tsirmpa I, et al. Tissue inhibitor of metalloproteinase- 2 as a multifunctional molecule of which the expression is associated with adverse prognosis of patients with urothelial bladder carcinomas. Clinical cancer research. 2003;9(15):5573-81.

20. Lamy S, Ouanouki A, Béliveau R, Desrosiers RR. Olive oil compounds inhibit vascular endothelial growth factor receptor-2 phosphorylation. Experimental cell research. 2014;322(1):89-98

21. Mărgăritescu C, Pirici D, Stîngă A, Simionescu C, Raica M, Mogoantă L, et al. VEGF expression and angiogenesis in oral squamous cell carcinoma: an immunohistochemical and morphometric study. Clinical and experimental medicine. 2010;10(4):209-14.

22. Naderi NJ, Tirgari F, Keshavarz Z. Vascular endothelial growth factor expression and vascular density in oral squamous cell carcinoma (OSCC): A study on clinical and histopathologic significance. Medical journal of the Islamic Republic of Iran. 2016;30:358.

23. Lalla RV, Boisoneau DS, Spiro JD, Kreutzer DL. Expression of vascular endothelial growth factor receptors on tumor cells in head and neck squamous cell carcinoma. Archives of Otolaryngology-Head \& Neck Surgery. 2003;129(8):882-8.
24. Papachristodoulou A, Tsoukala M, Benaki D, Kostidis S, Gioti K, Aligiannis N, Pratsinis H, Kletsas D, Skaltsounis A, Mikros E and Tenta R. Oleuropein is a powerful sensitizer of doxorubicin-mediated killing of prostate cancer cells and exerts its action via induction of autophagy. Journal of Cancer Research and Treatment. 2016; 4:61-8.

25. Chen W-L, Kuo K-T, Chou T-Y, Chen C-L, Wang C-H, Wei Y-H, et al. The role of cytochrome c oxidase subunit Va in non-small cell lung carcinoma cells: association with migration, invasion and prediction of distant metastasis. BMC cancer. 2012;12(1):273

26. Lipworth L, Martínez MaE, Angell J, Hsieh C-C, Trichopoulos D. Olive oil and human cancer: an assessment of the evidence. Preventive Medicine. 1997;26(2):181-90.

27. Mani SA, Guo W, Liao M-J, Eaton EN, Ayyanan A, Zhou AY, et al. The epithelial-mesenchymal transition generates cells with properties of stem cells. Cell. 2008; 133(4):704-15.

28. Rørth P. Collective guidance of collective cell migration. Trends in cell biology. 2007;17(12):575-9.

29. Harisi R, Kenessey I, Olah JN, Timar F, Babo I, Pogany G, et al. Differential inhibition of single and cluster type tumor cell migration. Anticancer research. 2009;29(8):2981-5.

30. George A, Ranganathan K, Rao UK. Expression of MMP-1 in histopathological different grades of oral squamous cell carcinoma and in normal buccal mucosa-an immunohistochemical study. Cancer Biomarkers. 2010; 7(6):275-83

31. Gialeli C, Theocharis AD, Karamanos NK. Roles of matrix metalloproteinases in cancer progression and their pharmacological targeting. The FEBS journal. 2011; 278(1):16-27.

32. Benbow U, Maitra R, Hamilton JW, Brinckerhoff CE. Selective modulation of collagenase 1 gene expression by the chemotherapeutic agent doxorubicin. Clinical cancer research. 1999;5(1):203-8. 